\title{
Leg Length Discrepancy to Influence on Kinematic Changes of the Pelvis and the Hip during Gait
}

\author{
MinSik Yong, SoHyun Park \\ Department of Physical Therapy, College of Health Sciences, Youngsan University, Yangsan, Korea
}

Purpose: The purpose of this study was to investigate the effects of leg length discrepancy on kinematic changes of the pelvis and hip during gait.

Methods: A total of ten healthy women with no history of neurological, musculoskeletal surgery or injuries, or pain in the lower limbs were recruited. They were assigned to two groups; the experimental group (LLD) consisting of five subjects leg length discrepancy of $10 \mathrm{~mm}$ to $18 \mathrm{~mm}$ and the control group (CON) consisting of five subjects leg length discrepancy of $<10 \mathrm{~mm}$. All participants were instructed to perform three walking trials for further analysis by using the Cortex 3.0 software program. Independent T-test and MannWhitney test were used to examine the effects of mild LLD on kinematic changes of the pelvis and hip during gait.

Results: Angles of hip flexion, hip abduction, pelvic obliquity, and pelvic tilt in the experimental group were not significantly different compared to those of the control group.

Conclusion: Mild leg length discrepancy induces kinematic changes in the lower limbs, including decreased hip flexion, increased hip abduction, and increased pelvic obliquity in the shorter limb, and increased hip adduction and increased pelvic obliquity in the longer limb. However, those changes were not significant.

Keywords: Leg length discrepancy, Motion analysis, Gait

\section{INTRODUCTION}

Leg length discrepancy (LLD), or leg length inequality, is defined as a condition in which the length of the lower extremities is remarkably unequal. ${ }^{1}$ LLD is a problem occurring in up to $70 \%$ of the population. Studies have shown that LLD of $2 \mathrm{~cm}$ or more affects at least one in every 1,000 people., ${ }^{2,3}$

LLD can be subdivided into two groups: structural LLD (SLLD) and functional LLD (FLLD). ${ }^{4}$ SLLD, also known as anatomic LLD, is defined as structural deformities resulting from differences in true bony leg length. It is an anatomical difference between the two lower extremities measured from the femoral head to the distal tibia and can be congenital or acquired. Congenital LLD can be induced by various abnormal developmental disorders. Causes of acquired LLD include paralysis, tumors, fractures, and surgical procedures, such as joint replacement. FLLD is defined as an asymmetrical leg

Received Dec 9, 2019 Revised Dec 24, 2019

Accepted Dec 26, 2019

Corresponding author SoHyun Park

E-mail ptpsh@ysu.ac.kr length caused by altered mechanics of the lower extremities, including muscle tightness or weakness, unilateral foot pronation or supination, knee hyperextension, and hip abduction/adduction tightness/contracture. ${ }^{1,5}$ Significant lower extremity malalignment may result from LLD. Because abnormal biomechanical factors are observed in a number of orthopedic disorders, it is thought that many pathological symptoms might be related to LLD. ${ }^{6-9}$ However, because several clinical factors found in orthopedic disorders can cause asymmetrical functional ability, it would be difficult to isolate and determine the effect of LLD on their function. ${ }^{10}$

There is disagreement regarding the magnitude of LLD warranting intervention. Several studies reported that LLD of $20 \mathrm{~mm}$ to 30 $\mathrm{mm}$ is the magnitude which may cause altered mechanics of the lower extremities. However, no clinically significant problems with LLD of less than $20 \mathrm{~mm}$ were identified. It was also reported that LLD of $5 \mathrm{~mm}$ to $9 \mathrm{~mm}$ seemed to be closely related to low back

Copylight (C2019 The Korean Society of Physical Therapy

This is an Open Access article distribute under the terms of the Creative Commons Attribution Non-commercial License (Http:// creativecommons.org/license/by-nc/4.o.) which permits unrestricted non-commercial use, distribution, and reproduction in any medium, provided the original work is properly cited. 
pain. ${ }^{11}$ In spite of these, only few studies have provided kinematic data of the lower extremities in subjects with FLLD of $10 \mathrm{~mm}$ to 18 $\mathrm{mm}$. Therefore, the purpose of this study was to investigate the effects of LLD of $\leq 18 \mathrm{~mm}$ (hereafter, mild LLD) on kinematic changes of the pelvis and hip during gait.

\section{METHOD}

\section{Study subjects}

A total of ten healthy women with no history of neurological, musculoskeletal surgery or injuries, or pain in the lower limbs were recruited. They were assigned to two groups, the experimental group (LLD) consisting of five subjects with leg length discrepancy of 10 $\mathrm{mm}$ to $18 \mathrm{~mm}$ and the control group $(\mathrm{CON})$ consisting of five subjects leg length discrepancy of $<10 \mathrm{~mm}$. All subjects had the right leg being shorter than the left. Leg length discrepancy was assessed by measuring the distance between the anterior superior iliac spine and the ipsilateral medial malleolus. The purpose and procedures of this study were explained to all subjects, and they provided written informed consent prior to their participation. The typical characteristics of the subjects are as follows (Table 1).

\section{Research procedure}

Three-dimensional motion analysis was performed using six cameras (Motion Analysis Corp., Santa Rosa, California) positioned at strategical locations to sample kinematic data at $60 \mathrm{~Hz}$. Fifteen reflective spherical markers were placed on the left and right anterior

Table 1. The characteristics of subjects

\begin{tabular}{lrr}
\hline Variable & CON $(n=5)$ & LLD $(n=5)$ \\
\hline Age $(\mathrm{yr})$ & $21.40 \pm 0.89$ & $21.60 \pm 0.89$ \\
Height $(\mathrm{cm})$ & $166.14 \pm 7.29$ & $167.46 \pm 7.87$ \\
Weight $(\mathrm{kg})$ & $58.37 \pm 7.82$ & $57.00 \pm 7.08$
\end{tabular}

CON: control group, LLD: experimental group, Values are reported as the Mean $\pm \mathrm{SD}$.

Table 2. Effects of leg length discrepancy on kinematic changes of the hip during mid stance phase and the whole stance phase

(unit: degree)

\begin{tabular}{|c|c|c|c|c|c|c|}
\hline & CON & LLD & \multirow{2}{*}{$\mathrm{p}$} & CON & LLD & \multirow[b]{2}{*}{$\mathrm{p}$} \\
\hline & \multicolumn{2}{|c|}{ RT } & & \multicolumn{2}{|c|}{ LT } & \\
\hline Hip flexion (mid stance) & $5.11 \pm 1.86$ & $3.94 \pm 1.86$ & 0.67 & $5.41 \pm 1.20$ & $5.39 \pm 2.09$ & 0.99 \\
\hline Hip adduction (mid stance) & $3.74 \pm 1.69$ & $3.11 \pm 0.50$ & 0.73 & $4.30 \pm 1.56$ & $5.96 \pm 0.66$ & 0.35 \\
\hline Hip flexion (whole stance) & $37.58 \pm 1.69$ & $36.52 \pm 0.90$ & 0.59 & $35.71 \pm 1.63$ & $36.19 \pm 0.80$ & 0.80 \\
\hline Hip adduction (whole stance) & $9.94 \pm 0.18$ & $8.09 \pm 1.05$ & 0.12 & $11.52 \pm 1.08$ & $9.61 \pm 0.73$ & 0.18 \\
\hline
\end{tabular}

CON: control group, LLD: experimental group, Values are reported as the Mean \pm SD.

superior iliac spines, midthighs, midshanks, lateral femoral epicondyles, lateral malleoli, second metatarsals, clacanei, and the sacrum based on the Helen Hayes marker set. Four additional markers were placed on the medial femoral condyles and the medial malleoli for calibration of the standing position (static data) only.

All participants were instructed to perform at least five walking trials for the practice. Participants walked at a self-selected speed. Then, three trials were recorded for further analysis by using the Cortex 3.0 software program (Motion Analysis Corp., USA).

These data were exported to the OrthoTrack 6.6.4 software (Motion Analysis Corp., USA), and three-dimensional joint angles for the hip, knee, and ankle were determined. Joint angle data were expressed in percentage of gait cycle was defined as the time period from initial contact to the following ipsilateral initial contact.

\section{Statistical analysis}

A regularity test was performed using the Shapiro-Wilk test method and all variables were normally distributed except left hip flexion and pelvic tilt. Independent T-test and Mann-Whitney test were used to examine the effects of mild LLD on kinematic changes of the pelvis and hip during gait. Statistical analyses were performed using SPSS ver. 18.0 (SPSS Inc., Chicago), and statistical significance was set at $\alpha=0.05$.

\section{RESULTS}

During mid stance phase, angles of hip flexion, hip abduction, pelvic obliquity, and pelvic tilt in LLD were not significantly different compared to those of CON ( $p>0.05$ ) (Tables 2, 3). During the whole stance phase, the range of angles of hip flexion, hip abduction, pelvic obliquity, and pelvic tilt in LLD were not significantly different compared to those of CON $(p>0.05)$. 
Table 3. Effects of leg length discrepancy on kinematic changes of the pelvis during mid stance phase and the whole stance phase

(unit: degree)

\begin{tabular}{lccc}
\hline & CON & LLD & $p$ \\
\hline Pelvic obliquity (mid stance) & $-0.72 \pm 0.77$ & $-0.93 \pm 0.62$ & 0.83 \\
Pelvic tilt (mid stance) & $7.31 \pm 2.17$ & $4.48 \pm 2.42$ & 0.41 \\
Pelvic obliquity (whole stance) & $7.78 \pm 0.78$ & $5.79 \pm 0.62$ & 0.08 \\
Pelvic tilt (whole stance) & $2.49 \pm 0.54$ & $2.45 \pm 0.45$ & 0.96 \\
\hline
\end{tabular}

CON: control group, LLD: experimental group, Values are reported as the Mean \pm SD.

\section{DISCUSSION}

A number of studies have indicated that LLD is associated with the occurrence of pathological conditions such as stress fractures, osteoarthritis, low back pain, and running injuries. ${ }^{12}$ However, there is still controversy regarding the magnitude of LLD necessary to warrant treatment. It has been reported that no significant symptoms were found in LLD of $10 \mathrm{~mm}$ to $25 \mathrm{~mm}$. Furthermore, from the results of several studies, there were no significant effects of LLD of $<10 \mathrm{~mm}$ on gait biomechanics. ${ }^{13}$ However, other studies demonstrated that LLD of $<10 \mathrm{~mm}$ influences not only the possibility of inducing osteoarthritis but also compensation in lower limb kinematics during gait. ${ }^{14}$

The present study measured the angles of the hip and pelvis to investigate the effects of mild LLD on lower limb kinematics during mid stance phase. Angles of hip flexion, hip abduction, pelvic obliquity, and pelvic tilt were slightly different between CON and LLD, but differences were not statistically significant $(\mathrm{p}>0.05)$.

It is widely accepted that many different compensatory strategies for LLD can occur in gait. To functionally lengthen the short limb, compensation mechanisms, including decreased hip flexion, increased hip abduction, and increased downward pelvic obliquity, can be used. ${ }^{10,15}$ During mid stance, those changes were observed in the short limb, but they were not significant $(\mathrm{p}>0.05)$. Compensatory mechanisms to shorten the longer limb including increased hip flexion, increased hip adduction, and increased upward pelvic obliquity can occur, but those changes were not significant either ( $p>0.05)$. The results of the present study suggest that mild LLD could not induce significant changes in lower limb kinematics which were associated with the occurrence of injuries in previous studies, demonstrating that there is no effect of LLD on pathological condition or lower limb kinematics. To make those results more obvious, the present study investigated the ranges of angles of hip flexion, hip abduction, pelvic obliquity, and pelvic tilt during stance phase.

The results showed that no significant differences were observed in either the longer or shorter limb during stance phase. There have been several studies showing that mild LLD causes kinematic compensation of the lower limbs leading to gait deviations. Two studies demonstrated that mild LLD induces biomechanical changes in the lower limbs and leads to asymmetrical gait deviations. It was also found that lower limb kinematic compensation associated with asymmetrical gait occurred with mild LLD. ${ }^{13,15}$ The most common strategy to compensate for mild LLD was the induction of pelvic obliquity. ${ }^{14}$ However, the present study showed greater differences in angles of hip flexion and abduction than in pelvic obliquity even though the differences were not significant.

In conclusion, the present study demonstrated that mild LLD induces kinematic changes in the lower limbs, including decreased hip flexion, increased hip abduction, and increased pelvic obliquity in the shorter limb, and increased hip adduction and increased pelvic obliquity in the longer limb. However, those changes were not significant. The results were not in accordance with previous studies which demonstrated that mild LLD can cause significant change in the lower limbs. Because the present study did not identify kinematic gait deviations of the knee, ankle, or foot able to be caused by mild LLD, the potential impact of mild LLD should not be overlooked.

The limitations of this study are that it is difficult to generalize because of small sample size and the fact that all the participants were healthy women. In addition, only changes of angle in the pelvis and hip were provided. Therefore, it is suggested that further study is needed to consider factors, including internal moments, muscle activity of the lower limbs, and knee, ankle, and foot kinematics, which may be affected by LLD.

\section{ACKNOWLEDGEMENTS}

This work was supported by Youngsan University Research Fund of 2018. 


\section{REFERENCES}

1. Gurney B. Leg length discrepancy. Gait \& posture. 2002;15(2):195-206.

2. Woerman AL, Binder-Macleod SA. Leg length discrepancy assessment: Accuracy and precision in five clinical methods of evaluation. J Orthop Sports Phys Ther. 1984;5(5):230-9.

3. Guichet J-M, Spivak JM, Trouilloud P et al. Lower limb-length discrepancy. An epidemiologic study. Clin Orthop Relat Res. 1991(272):235-41.

4. Danbert RJ. Clinical assessment and treatment of leg length inequalities. J Manipulative Physiol Ther. 1988;11(4):290-5.

5. Blake RL, Ferguson H. Limb length discrepancies. J Am Podiatr Med Assoc. 1992;82(1):33-8.

6. Fujimaki H, Inaba Y, Kobayashi $\mathrm{N}$ et al. Leg length discrepancy and lower limb alignment after total hip arthroplasty in unilateral hip osteoarthritis patients. J Orthop Sci. 2013;18(6):969-76.

7. Sanhudo JA, Gomes JL. Association between leg length discrepancy and posterior tibial tendon dysfunction. Foot Ankle Spec. 2014;7(2):119-26.

8. Harvey WF, Yang M, Cooke TD et al. Association of leg-length inequality with knee osteoarthritis: a cohort study. Ann Intern Med. 2010;152(5): 287-95.
9. Khamis S, Leisman G, Carmeli E. Detecting the presence of leg length discrepancy based on gait deviations and functional measurement of leg length during walking. BMJ Case Rep. 2017;2017.

10. Khamis S, Carmeli E. Relationship and significance of gait deviations associated with limb length discrepancy: a systematic review. Gait Posture. 2017;57:115-23.

11. Young RS, Andrew PD, Cummings GS. Effect of simulating leg length inequality on pelvic torsion and trunk mobility. Gait \& posture. 2000; 11(3):217-23.

12. Gurney B, Mermier C, Robergs R et al. Effects of limb-length discrepancy on gait economy and lower-extremity muscle activity in older adults. JBJS. 2001;83(6):907-15.

13. Khamis S, Carmeli E. The effect of simulated leg length discrepancy on lower limb biomechanics during gait. Gait Posture. 2017.

14. Walsh M, Connolly P, Jenkinson A et al. Leg length discrepancy-an experimental study of compensatory changes in three dimensions using gait analysis. Gait Posture. 2000;12(2):156-61.

15. Resende RA, Kirkwood RN, Deluzio KJ et al. Biomechanical strategies implemented to compensate for mild leg length discrepancy during gait. Gait Posture. 2016;46:147-53. 